\title{
Performance curve of basic skills in single-incision laparoscopy versus conventional laparoscopy: Is it really more difficult for the novice?
}

\author{
S. A. F. Fransen - L. S. Mertens • S. M. B. I. Botden • \\ L. P. S. Stassen • N. D. Bouvy
}

Received: 5 June 2011/Accepted: 20 October 2011/Published online: 20 November 2011

(C) The Author(s) 2011. This article is published with open access at Springerlink.com

\begin{abstract}
Background Single-incision laparoscopy is a step forward toward nearly scarless surgery. Concern has been raised that single-incision laparoscopy is technically more challenging than conventional laparoscopy. This study researched the performance curves of novice trainees for single-incision laparoscopy (SILS) versus conventional laparoscopy for performing two basic tasks on a box trainer.

Methods In this study, 20 novice participants performed two tasks (peg transfer and a dissection task) on a standard box trainer. All the participants practiced each task 11 times and were randomized in two groups. The first group performed the tasks on a box trainer through three incisions. The second group used a single-incision access with the same box trainer. The assessment scores for errors and time were recorded. The 2nd, 8th, and 11th runs of both tasks in both settings were assessed to objectify the gain in basic laparoscopic skills.

Results The performance curves for both groups improved significantly in terms of both time and errors in performing the two tasks $[P<0.01$, analysis of variance (ANOVA)]. For the first task, no significant difference in time between the two groups was observed at the top of the performance curve (mean, $212 \pm 64$ vs. $182 \pm 48 \mathrm{~s}$ ), but the SILS group performed with fewer errors $(1.3 \pm 1.5 \mathrm{vs}$. $2.7 \pm 2.11)$. However, the difference was not significant
\end{abstract}

Presented at the 19th EAES Congress, June 15-18, 2011, Torino, Italy.

S. A. F. Fransen $(\bowtie) \cdot$ L. S. Mertens - S. M. B. I. Botden ·

L. P. S. Stassen - N. D. Bouvy

Department of Surgery, Maastricht University Medical Centre,

P. Debyelaan 25, 6229 HX Maastricht, The Netherlands

e-mail: fransensofie@hotmail.com
$(P=0.10)$. The dissection task was performed faster in the SILS group (mean, $205 \pm 78$ vs. $243 \pm 40 \mathrm{~s} ; P=0,18$ ) with fewer errors $(0.7 \pm 1.05$ vs. $1.9 \pm 2.1 ; P=0.12)$, but the difference was not significant.

Conclusion This study showed a significant improvement in basic skills for both the SILS and conventional laparoscopy settings after 11 repetitions. These data suggest that box training shows no significant difference between conventional laparoscopic and single-incision laparoscopic settings in terms of error or time in performing basic tasks at a low complexity level for the novice. These data also show significant improvement in basic skills over a relatively short period.

Keywords G-I · Human/robotic - Training/courses · Surgical

Laparoscopic surgery is widely accepted and applied for many surgical procedures and has profoundly altered the armamentarium available to the individual surgeon. It requires surgeons to operate with a two-dimensional image of the operating field and with only the tips of the instruments visible. Also, an extra difficulty is the "fulcrum effect," in which the movements of the surgeon's hand results in contrary deflections of the working end of a laparoscopic instrument, creating a disparity between visual and proprioceptive feedback [1-3].

The tendency to minimize surgical trauma encourages the use of new approaches in laparoscopic surgery. Currently, single-incision laparoscopic surgery (SILS) is applied in several surgical procedures such as cholecystectomy, appendectomy, and gastric sleeve resection. This single-incision technique has come to be known by a variety of names and acronyms including SILS, LESS, 
SPA, SSL, OPUS, E-NOTES, TUES, NOTUS, SLAPP, SPLS, SPL, SLIT, and SIMPL [4]. For the purpose of this study, we refer to the technique by the commonly used term, single-incision laparoscopic surgery (SILS).

The SILS approach is considered a step forward toward nearly scarless surgery. With SILS, the approach to operating on the target organs remains the same, but the access to the abdomen is obtained differently. Cholecystectomy performed in the conventional laparoscopic setting uses four small incisions, whereas in the SILS setting, all working instruments enter the abdomen through one umbilical incision. Difficulties to be expected with the single-incision technique are a lack of triangulation due to parallel entry of the working instruments, clashing of the instruments, and decreased visualization or exposure [5].

Concerns have been raised that SILS is more technically challenging than conventional laparoscopy. The difficulty of the performance curve with a new technique has led many surgeons to question the applicability and advisability of using newly developed laparoscopic techniques such as the single-incision laparoscopy. Measuring technical performance on a surgical simulator in the laboratory setting allows for the use of standardized tasks and validated metrics in a controlled and safe environment.

The performance curves with box trainers for conventional laparoscopic procedures have been described previously [2, 6-9], but performance curves for single-incision basic laparoscopic skills have not been published to date. This study therefore aimed to compare the performance curves for gaining bimanual coordination skills between single-incision laparoscopy and conventional laparoscopy performed by novice participants on box trainers.

\section{Methods}

Subjects

The participants suitable for this study were novices with no laparoscopic experience either in the operating room or with simulator training. All the participants were medical interns during their surgical rotation or after its completion. We recruited them at the Surgical Department of the Maastricht University Medical Centre, Maastricht, the Netherlands. The study was completed by 20 participants.

\section{Equipment}

For the abstract environment of the abdomen, a standard box trainer (Endo Innovation ${ }^{\mathrm{TM}}$, 's Hertogenbosch, The Netherlands) was used. The first group used the LESS port (Olympus, Hamburg, Germany) to introduce the instruments into the abdomen in the SILS setting. This port consists of three gel ports: one for the camera and two for the instruments. The port was introduced using a standardized $3-\mathrm{cm}$ incision positioned between the previous conventional port incisions. In this setting, double-curved instruments (Olympus, Hamburg, Germany) were used. The second group used three 5- to $12 \mathrm{~mm}$ working ports (Covidien, Dublin, Ireland) in a conventional laparoscopy setting with straight instruments (Olympus).

The Endo-Eye laparoscopic camera (5 mm; Olympus) was connected to a screen placed at an ergonomic height and location for the study participants. The inserts for the tasks, handmade by the researchers, were reusable to create a standardized situation (Figs. 1,2).

\section{Settings}

The participants were randomly assigned to two groups: the SILS group and the conventional laparoscopy group. Both groups performed two basic tasks [4] (peg transfer and dissection) based on the fundamentals of laparoscopic surgery. Performance was measured by time for completion and errors. Each participant performed both tasks 11 times spread over 2 days. All the tasks were observed by two observers, who carefully assessed the tasks for errors and time.

\section{Task 1: Peg transfer}

A series of six iron rings had to be transferred from the right to the left pins on the tray in the box. The rings were

\section{Box trainer, setup single-port}

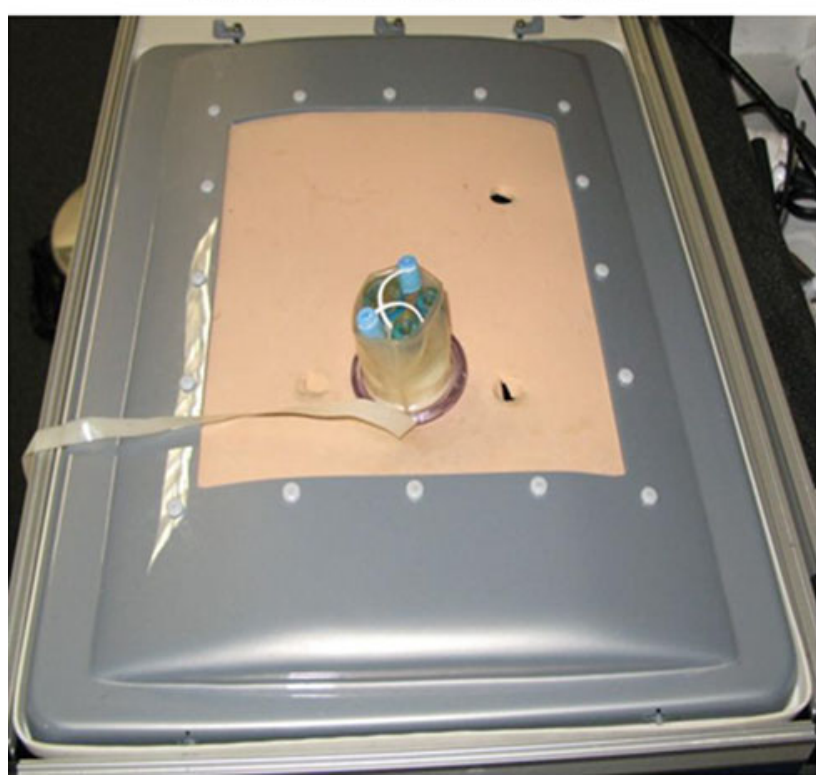

Fig. 1 Setup box trainer: single-incision laparoscopic surgery (SILS) setting 

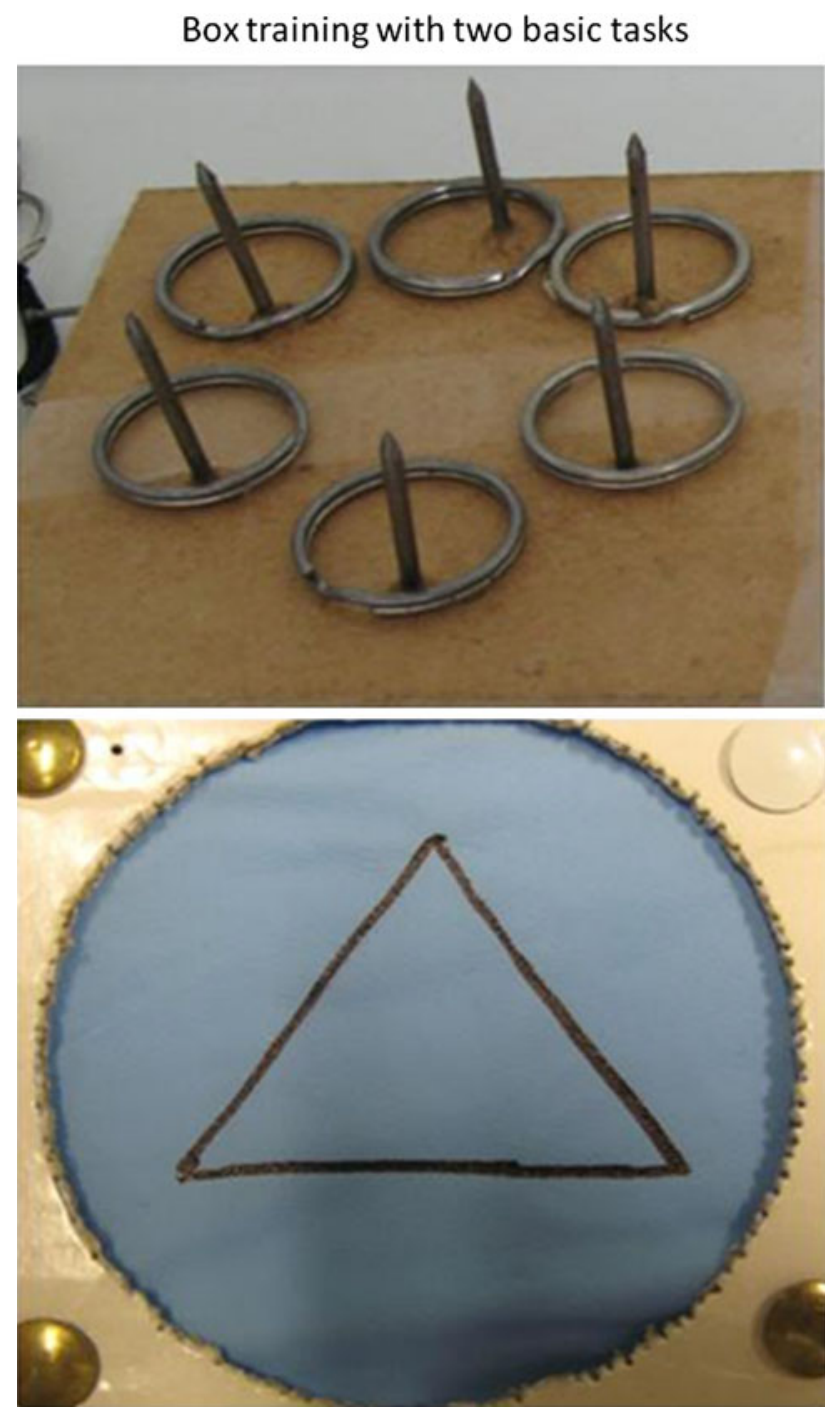

Fig. 2 Box trainer: tasks 1 and 2

picked up by the right dissection clamp, transferred in the air to the left instrument, and placed on the left side of the field. The participants then had to transfer the rings from the left to the right pins in the same manner. This exercise was scored by time for completion (with a cut offtime of $600 \mathrm{~s}$ ) and by penalty scores for dropping of the ring or inability to transfer a ring.

\section{Task 2: Dissection}

The purpose of this task was to dissect a circle out of a double-layer polyurethane glove. On this glove, two circles were drawn: an inner circle and an outer circle. The distance between the two margins was $0.5 \mathrm{~cm}$. Dissection had to be performed in the space between the two circles. Only the upper layer of the double-layer glove had to be dissected. The glove was placed at an ergonomic distance inside the box trainer at a $45^{\circ}$ angle. A dissection clamp and scissors were used for this task. The scores were determined by time for completion (with a maximum of $600 \mathrm{~s}$ ) and penalty scores. The dissected part was analyzed, and an error was scored each time the dissection was performed through the circles.

Protocol

The 20 participants were randomly assigned to the two groups. Each participant performed the trainings individually. The investigators informed the participants on both tasks. The participants received the opportunity to exercise with the instruments outside the box to become familiar with the functions of the instruments.

Both groups started with the first task (peg transfer), which was repeated six times the first day. After completion of this repetition, the second task (dissection) was performed and also repeated six times. Between the repetitions, breaks were introduced to avoid fatigue and frustrations. The second day, both tasks were repeated five times in the same manner.

The first run of both tasks in both settings was excluded from our results to avoid the bias of unfamiliarity with both the task and the setting. The 2nd, 8th (top of the performance curve), and 11th (last) runs of both tasks were assessed. During the training, the assessment scores of the assessment module were obtained on a standardized evaluation form (Fig. 3).

\section{Statistical analysis}

All data were processed and analyzed using the Statistical Package for the Social Sciences, (SPSS version 16.0; SPSS, Chicago, IL, USA). Total time and errors were compared between the two settings using a single analysis of variance (ANOVA) model. The differences between the performance scores at the 2nd, 8th, and 11th repetitions were calculated with the independent paired $t$-test. No power analysis was performed because this was a pilot study.

\section{Results}

Demographics

The mean age of the participants was 24 years. Of the 20 participants ( 8 women and 12 men), 19 were right-handed.

Task 1: Peg transfer

The performance curve for both the SILS and the conventional laparoscopic settings shows significant improvement in time and errors $(P<0.01$, ANOVA). 
Fig. 3 Outline of the protocol

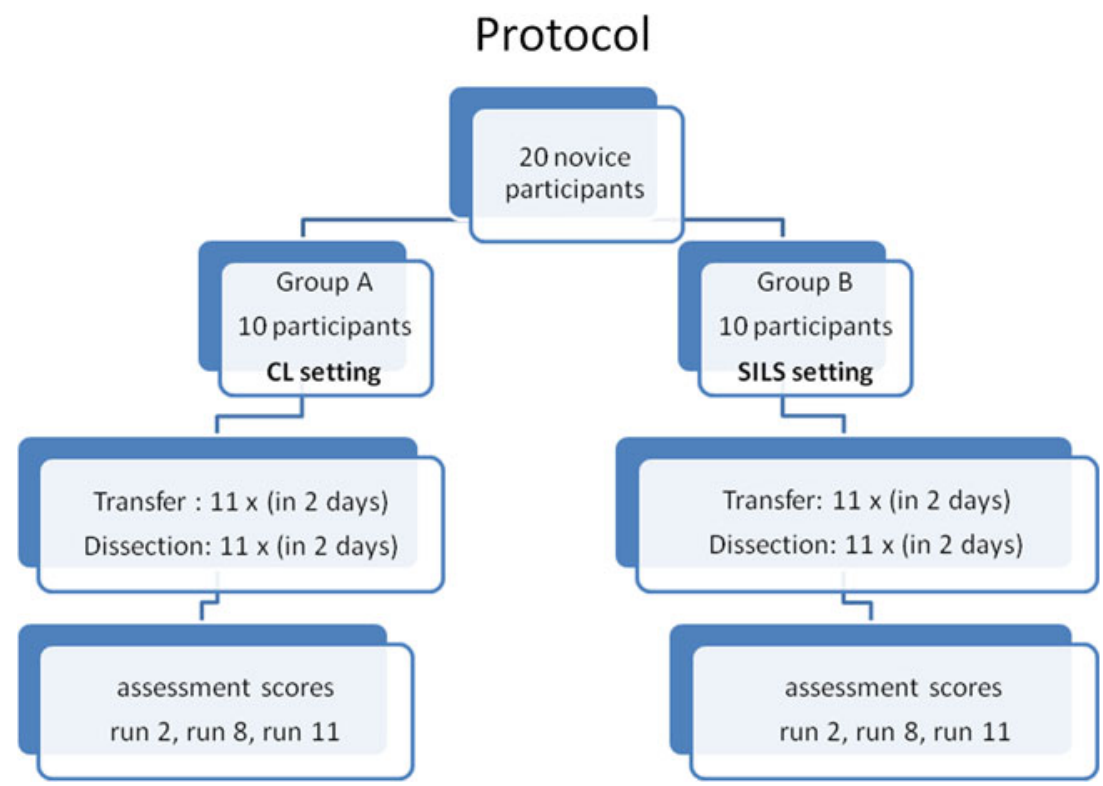

Table 1 Gaining proficiency in two basic tasks: comparing runs 2, 8 and 11

\begin{tabular}{|c|c|c|c|c|c|}
\hline Tasks & Run & Score & $\begin{array}{l}\text { Conventional group } \\
\text { (mean } \pm \text { SD) }\end{array}$ & $\begin{array}{l}\text { SILS group } \\
(\text { mean } \pm \mathrm{SD})\end{array}$ & $P$ value $(d f)$ \\
\hline \multirow[t]{6}{*}{1 (translocation of rings) } & \multirow[t]{2}{*}{2} & Time & $337 \pm 116$ & $349 \pm 122$ & $>0.82(17,91)$ \\
\hline & & Errors & $2.5 \pm 1.1$ & $3.1 \pm 2.0$ & $>0.44(14,24)$ \\
\hline & \multirow[t]{2}{*}{8} & Time & $182 \pm 48$ & $212 \pm 64$ & $>0.25(16,71)$ \\
\hline & & Errors & $2.7 \pm 2.11$ & $1.3 \pm 1.5$ & $>0.11(16,64)$ \\
\hline & \multirow[t]{2}{*}{11} & Time & $192 \pm 49$ & $229 \pm 145$ & $>0.45(11,03)$ \\
\hline & & Errors & $2.0 \pm 2.3$ & $1.3 \pm 1.7$ & $>0.45(16,84)$ \\
\hline \multirow[t]{6}{*}{2 (dissection of tissue) } & \multirow[t]{2}{*}{2} & Time & $327 \pm 81$ & $296 \pm 97$ & $>0.46(17,45)$ \\
\hline & & Errors & $2.3 \pm 1.7$ & $1.5 \pm 1.35$ & $>0.27(16,86)$ \\
\hline & \multirow[t]{2}{*}{8} & Time & $243 \pm 40.4$ & $205.5 \pm 68.38$ & $>0.19(13,48)$ \\
\hline & & Errors & $1.9 \pm 1.13$ & $0.7 \pm 1.05$ & $>0.13(13,19)$ \\
\hline & \multirow[t]{2}{*}{11} & Time & $201 \pm 55.37$ & $178.6 \pm 63.14$ & $>0.41(17,69)$ \\
\hline & & errors & $0.6 \pm 0.96$ & $0.8 \pm 0.91$ & $>0.64(17,95)$ \\
\hline
\end{tabular}

SILS Single-incision laparoscopic surgery, SD standard deviation

Table 1 shows the detailed performance of both groups at the 2nd, 8th, and 11th runs. Comparison of these runs shows that the peg transfer was performed faster in the laparoscopy setting, but the difference was not significant (Table 1). The penalty scores were lower in the laparoscopy setting during the second run. At the 8th and 11th runs, the penalty scores were lower for the SILS setting.

For the SILS group, the top of the performance curve for errors was reached at the eighth run (Table 1). For the conventional group, the top of performance was reached at the seventh run. At this top of the performance curve, we observe fewer errors in the SILS group than in the conventional laparoscopic group, although the difference is not significant. The conventional group performed faster but not significantly faster. Task time decreased significantly between the second and the eighth runs in both settings ( $P<0.05$ for both groups). Only the SILS group performed better in terms of errors between the second and eighth runs. Comparison of the translocation task between the top of performance and the 11th run shows that time did not improve significantly in either group. No significant improvement in errors occurred in either setting (Figs. 4, 5).

Task 2: Dissection task

The performance curve for the second task showed significant improvement ( $p<0.01$, ANOVA) in both groups 


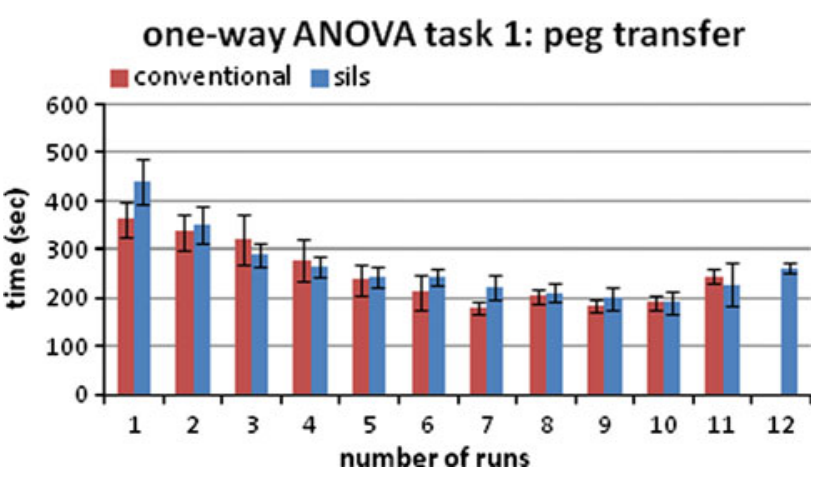

Fig. 4 Task 1. Peg transfer: time versus repetition

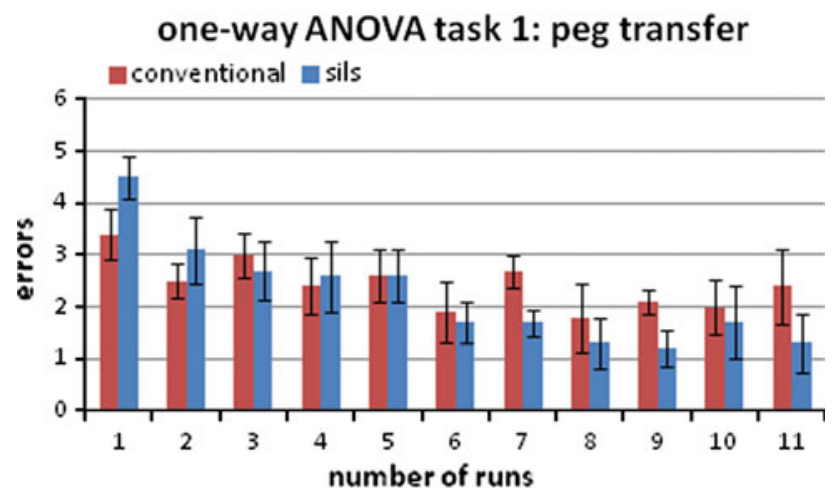

Fig. 5 Task 1. Peg transfer: errors versus repetition

for time and errors. The dissection task was performed faster in the SILS setting, as shown when the 2nd, 8th, and 11th runs of the SILS setting were compared with those of the conventional laparoscopy setting, although the difference was not significant. Fewer errors occurred in the SILS setting during the second and eighth runs, but for the final run, the laparoscopy setting showed fewer errors (Table 1). However, no significant differences for any of the runs were observed.

In both settings, comparison of the scoring for time shows that a plateau was reached at the third repetition (Fig. 6). In the SILS setting, the top of the performance score for errors was at the eighth repetition (Fig. 7). In the conventional group, this top was reached at the sixth repetition (Fig. 7). At the eighth repetition, the SILS group performed faster than the conventional group with fewer errors, but the difference was not significant (Table 1). Comparison of the baseline scores with the scores at the top of the performance curve showed a significant difference between the times in the two groups. Neither group showed significant improvement for errors at the top of the performance curve. Comparison of the eighth run with the last run showed no significant improvement for time or errors in either setting.

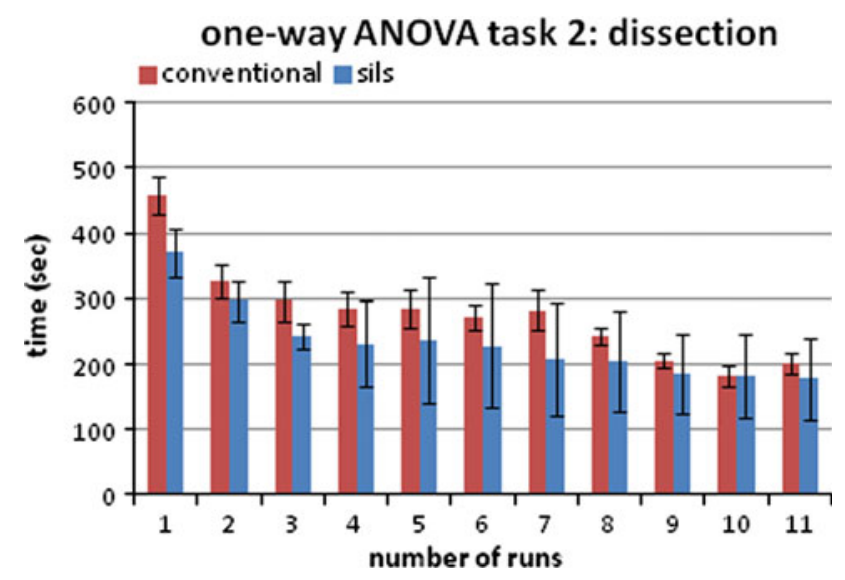

Fig. 6 Task 2. Dissection: time versus repetition

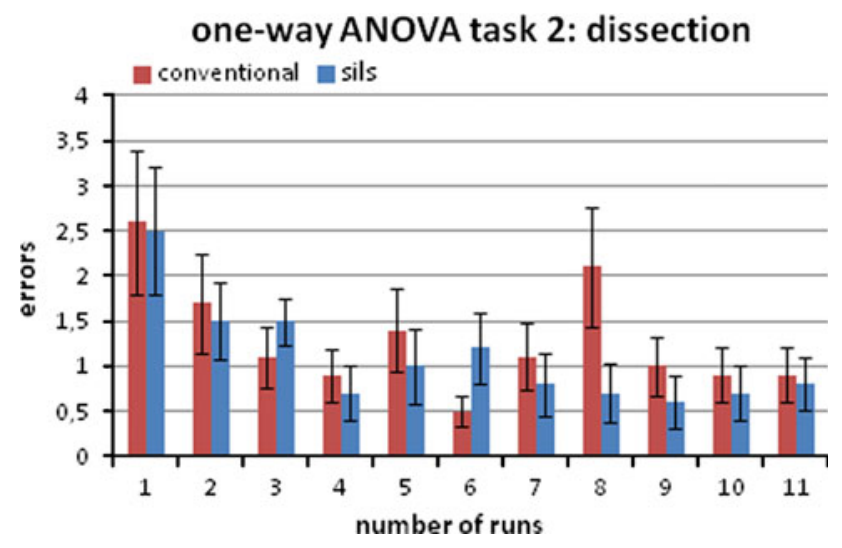

Fig. 7 Task 2. Dissection: errors versus repetition

\section{Discussion}

The SILS approach might be limited by its technical constraints including clashing of the instruments and limited operation space. Therefore, it is assumed that SILS is more technically challenging than conventional laparoscopy.

This study, with a relative small sample size, demonstrated that the participants significantly improved in both time and errors during the single-incision laparoscopic surgery (SILS) and conventional laparoscopy settings in both basic tasks: The final scores in both groups improved significantly compared with baseline scores. The performance in terms of time for both tasks showed that a plateau was reached after an equal number of runs in both settings. The performance in terms of errors showed that the SILS setting required two more trials before the top of performance was reached. However at this top for the SILS group, fewer errors were scored within less time than for the conventional group. The authors acknowledge that for this small number of participants, considerable differences in standard deviations are noticed (Table 1). Therefore, it 
will be interesting in the near future to expand the number of participants in each group to evaluate the significance of this difference.

If single-incision laparoscopy becomes a widespread clinical phenomenon, it will be necessary for current and future surgeons to master the skills of single-incision laparoscopy. More complex tasks may be necessary for accurate practice of the skills. To gain proficiency in laparoscopic skills, surgeons-in-training must experience their own learning curve [7].

In the beginning of our training, we noticed that trainees were more directed by time movement than by errors. A plateau for time was reached faster without improvement for errors. This implies that the performance curve for surgical speed was shorter than that for surgical errors. For future and more complex tasks, video recording might be necessary to assess the outcome for the tasks at a higher complexity level.

Analysis of the performance curves for both tasks and both settings shows dips at the fourth, fifth, and seventh runs (Figs. 4, 5, 6, 7). Practicing too intensely in 1 day may cause exhaustion and could negatively influence performance. We hypothesize that this explains the dips at the fourth and fifth runs. Run seven was the first run on the second day, during which the participants had to get used to the technique again. This phenomenon also is seen in other performance curve studies [6].

Comparison of the differences between the trainees for each setting and each task showed that the trainees attained similar levels (Table 1). A relation exists between cognitive abilities and skills acquisition in the early phase of learning new skills, but this correlation seems to decline when the procedures becomes more routine. An explanation for the decline in performance after the eighth run can be based on the fact that the tasks became routine and the participants were less focused and motivated to perform their best $[6,7]$.

For the top of the performance curve, we focused on the eighth repetition. Comparison of the top of the curve between the two groups showed fewer errors in the SILS groups, but the difference was not significant. This lack of significant differences between the two groups is a remarkable finding because it may indicate that both techniques performed on box trainers are comparable in terms of the learning curve. The authors acknowledge, however, that the number of participants in this study was very small. Because this study was a pilot study, no sample power calculation was performed.

Given the large standard differences between the individual scores and the small group sizes, we additionally performed the Mann-Whitney $U$ test. We have not described these outcomes, but the results were comparable.
Expanding the number of participants may be interesting for future observations, and statistical significance might be achieved.

Single-incision laparoscopy may have restrictions such as lack of triangulation and clashing of instruments. The setup of the experiment in this study was very different from the real life. Therefore, caution should be observed in drawing conclusions from these data for the real-life situation. We used experiments only in a box setting. Additionally, the two performed basic tasks were of a very low difficulty level.

\section{Conclusion}

Single-incision laparoscopy may seem more technically challenging than conventional laparoscopic surgery. However, the data analyzed in this study suggest that box training resulted in no significant performance differences in terms of error or time for the basic tasks at a low complexity level on the part of the novice in conventional laparoscopic and single-incision laparoscopic settings. These data also show significant improvement in basic skills over a relatively short period. Both groups became familiar with the specific psychomotor skills in each setting after repeated practice of the standardized tasks.

Disclosures S. A. F. Fransen, L. S. Mertens, S. M. B. I. Botden, L. P. S. Stassen, and N. D. Bouvy have no conflicts of interest or financial ties to disclose.

Open Access This article is distributed under the terms of the Creative Commons Attribution Noncommercial License which permits any noncommercial use, distribution, and reproduction in any medium, provided the original author(s) and source are credited.

\section{References}

1. Chamberlain RS, Sakpal SV (2009) A comprehensive review of single-incision laparoscopic surgery and natural orifice transluminal endoscopic surgery techniques for cholecystectomy. J Gastrointest Surg 13:1733-1740

2. Smith CD, Farrell TM (2001) Assessing laparoscopic manipulative skills. Am J Surg 181:547-550

3. Gallagher AG, Mc Clure N, Mc Guigan J et al (1998) An ergonomic analysis of the fulcrum effect in the acquisition of endoscopic skills. Endoscopy 30:617-620

4. Santos BF, Enter D, Soper NJ (2011) Single-incision laparoscopic surgery versus standard laparoscopic surgery: a comparison of performance using a surgical simulator. Surg Endosc 25:483-490

5. Chow A, Purkayastha S, Aziz O, Paraskeva P (2010) Singleincision laparoscopic surgery for cholecystectomy: an evolving technique. Surg Endosc 24:709-714

6. Botden SMBI, de Hingh IHJT, Jakimowicz JJ (2009) Suturing training in augmented reality: gaining proficiency in suturing skills faster. Surg Endosc 23:2131-2137 
7. Van Sickle KR (2005) Construct validation of the ProMIS simulator using a novel laparoscopic suturing task. Surg Endosc 19:1227-1231

8. Schijven MP, Jakimowicz JJ (2004) The learning curve on the Xitact LS 500 laparoscopy simulator: profiles of performance. Surg Endosc 18:121-127
9. Aggarwal R, Moorthy K, Darzi A (2004) Laparoscopic skills training and assessment. Br J Surg 91:1549-1558 